\title{
Parallel Newton-Krylov-Schwarz Method for Solving the Anisotropic Bidomain Equations from the Excitation of the Heart Model
}

\author{
Maria Murillo and Xiao-Chuan Cai \\ Computer Science Department \\ University of Colorado \\ Boulder, CO 80302 \\ (murillo, cai)@colorado.edu
}

\begin{abstract}
We present our preliminary results from applying the Newton-KrylovSchwarz method for the simulation of the electrical activity of the heart in two dimensions. We use the bidomain nonlinear equations, using a fully implicit time discretization scheme, and solving the resulting large system of equations with a Newton based algorithm at each step. We incorporate anisotropy into our model, and compare the results of using various conductivity ratios between the intra-cellular and extra-cellular domains. We also compare our results with previous work that has been done using less precise techniques, such as explicit and semi-implicit schemes, and less detailed models, such as the Monodomain model, and isotropic as well as quasi-isotropic Bidomain models. The results are obtained using PETSc of the Argonne National Laboratory.
\end{abstract}

\section{Introduction}

The rapid increase on heart failure related deaths and other problems prompted the need for a better understanding of all processes involved in the heart system, plus the immediate need to determine mechanisms to either prevent or alleviate these problems. Researchers are now trying to extract more and better data, and to determine better and more complex models which can explain the data. In recent issues of SIAM News, Quarteroni [] has presented a very concise discussion of the current state with respect to modeling the blood circulation in the 
heart. In these two articles, Quarteroni also mentions the importance of having a more comprehensive model of the heart system, including the fluid dynamics, the structure, and the electrical signals that activate the process, as well as the various biochemical reactions that are believed to be responsible for many of these interactions. Of all these important components, we concentrate on this paper on the treatment of the electrical activity of the heart. The most recent, and advanced model explains the electrical mechanisms in the heart system by a bidomain model, where an intracellular medium is separated from an extracellular medium by a membrane, and it is across this membrane that the critical processes take place. Once we have a representative model of this electrical activity, we are faced with the challenge of working with this model in a discrete and numerical approach; as Quarteroni mentions, even though great progress has been made in this area, we still need to move forward and develop and effectively implement more accurate techniques that preserve most of the details and accuracy of the given models. Among such techniques, the fully implicit methods are proven to be very accurate, although costly. Incorporating domain decomposition techniques along with these accurate methods, allows us to take advantage of today's parallel supercomputing powers making the solution of the associated systems feasible in time and space. Here we present the preliminary results of incorporating various degrees of anisotropy into this Bidomain model, and solving the set of time-dependent nonlinear equations with a fully implicit Newton-Krylov-Schwarz scheme.

\section{The Model}

\subsection{Bidomain Model}

We consider here the Bidomain model which includes three distinct areas within the heart tissue: the intracellular domain, the extracellular domain, and a membrane which separates the two previous ones. Originally, each one of these sections was s considered to be homogeneous, with all its properties being just averages.

This so-called bidomain model is the most accepted today, with slight variations to its complexity. The electrical activity of the heart is believed to be originated by changes in potential across these areas, which in turn, generate waveforms that propagate through the different cells [] . At the intracellular level, it is thought that enzyme activity is largely responsible for the generation of electrical fields at various phase boundaries within the cell. In the transmembrane, an action potential exists due to the effect of changes in ionic permeability and subsequent voltage differences. The extracellular electric field is represented by a waveform which apparently is directly correlated to the actions taking place at the membrane as well as the intracellular area; and, it appears to correspond to the outward and inward flows generated by changes in potential. The bidomain 
equations [?] can then be expressed as a reaction- diffusion system which consists of a nonlinear parabolic equation in $V$ coupled with an elliptic equation in the extracellular potential $V_{e}$

$$
\left\{\begin{array}{c}
\chi\left(C_{m} \frac{\partial V}{\partial t}\right)+I_{i o n}-I_{a p p}=-\nabla\left(D_{e} \nabla V_{e}\right) \\
\nabla\left(D_{i} \nabla V\right)=-\nabla\left(D \nabla V_{e}\right)
\end{array},\right.
$$

where $\chi$ and $C_{m}$ are constants, $I_{i o n}$ is the ionic current, and $I_{a p p}$ is a current applied to the system. The matrices $D$ refer to the conductivity tensors.

\subsection{The Ionic Current}

The bidomain equations are used to represent the propagation of the waveform generated by changes in potential. This is uncorrelated to the model being used to represent the internal ionic currents. Some work is being done in trying to understand these currents, how they generate and propagate. DiFrancesco and Noble [5] presented a very complex system with about a dozen of coupled nonlinear equations, and about 50 parameters.

One attempt to simplify even more the expression for this ionic current term was developed by using the Fitzhugh-Nagumo equations [], where only two variables are considered. One other model yet more simplistic than the previous, was proposed by Walton and Fozzard [?]. This model expresses the ionic current as dependent only on $V$. Although very simplified and tractable, this model is considered to still retain the main characteristics of the cardiac activity without including the smaller details. The ionic current is then expressed as:

$$
I_{\text {ion }}=A V\left(1-\frac{V}{V_{t}}\right)\left(1-\frac{V}{V_{p}}\right)
$$

where $\mathrm{A}$ is a scaling factor, and $V_{t}$ and $V_{p}$ are the potential threshold (where activation occurs) and the plateau value, respectively. For simplicity, this latter model for the Ionic current is the one we will be using for our experiments.

\subsection{Anisotropy}

An important issue to determine an appropriate model for the cardiac activity is the conductivity tensor $D$. Depending on the structure of this matrix, we are incorporating various degrees of anisotropy to the system. The simplest one would be a diagonal matrix, which indicates that conductivity varies only along the coordinate system. A more complex system requires the inclusion of a full matrix where the conductivity varies in every direction. An associated element is the fact that in the bidomain model, we have different degrees of anisotropy 
in the intracellular and extracellular media. The anisotropy comes from the fact that the heart is made up of fibers, and conductance is more readily in the direction of these fibers than on the transverse direction.

In this work, we assume our model is two-dimensional and the fiber is oriented in the direction of the axes; this implies we have the following structure for the conductivity tensors in the intracellular and extracellular tissue portions, $D_{i}$ and $D_{e}$ respectively:

$$
\begin{aligned}
& D_{i}=\left[\begin{array}{ll}
\sigma_{l}^{i} & \\
& \sigma_{t}^{i}
\end{array}\right], \\
& D_{e}=\left[\begin{array}{ll}
\sigma_{l}^{e} & \\
& \sigma_{t}^{e}
\end{array}\right],
\end{aligned}
$$

where the superscripts ${ }^{e}$ and ${ }^{i}$ refer to the mentioned extra and intracellular domains, and the subscripts $l$ and $t$ refer to the longitudinal and transverse directions where we are considering the existence of anisotropy.

Greater simplification can come from the anisotropy of the model; if we consider this two-dimensional case (for simplicity) and assume different conductivity values in the longitudinal as well as transversal direction only, but also assuming that the value of these anisotropy ratios is the same for both the intracellular, and the extracellular domain, then we refer to this as the quasi-isotropic model.

The bidomain model then simplifies when we assume the electrical potential on the interstitial region is zero (Monodomain model), or when the conductivity tensors are just a multiple constant of each other (isotropic or quasi-isotropic Bidomain model) [6].

\subsection{Boundary Conditions}

For the bidomain model, it is commonly assumed that there is no flux across the boundaries [1]. The reason being that since the potential $V$ seems to have constant values before and behind the exciting front, we assume it is not affected by the outside medium [?].

In particular, for our simplified model, this boundary condition can be expressed as

$$
\left\{\begin{array}{l}
\mathbf{n}^{\mathbf{T}} \mathbf{D}_{\mathbf{i}} \nabla \mathbf{V}=\mathbf{0} \\
\mathbf{n}^{\mathbf{T}} \mathbf{D} \nabla \mathbf{V}_{\mathbf{e}}=\mathbf{0}
\end{array}\right.
$$




\section{Numerical Approach}

There have been numerous attempts to numerically solve for the heart excitation problem. There are three major approaches: explicit, semi-implicit and implicit methods. In the case of the bidomain equations, more emphasis has been placed on the explicit and semi-implicit methods, since these methods are more easily tractable when implemented on a computer. However, there are many limitations associated with these methods, such as stability problems, step size limits in time, and others.

Hooke et al. [6] presented a general version of algebraic transformations that have been applied to the bidomain equations. The explicit Euler's method was the choice for the time integration. In Veronese and Othmer [14], a hybrid Alternating Direction Implicit (ADI) scheme is used for the nonlinear parabolic equation.

Bogar [1] extended the use of a semi-implicit method for the bidomain model, which has previously been used only for the Monodomain case. The approach is to split the nonlinear term into two parts: one is resolved implicitly (the resulting linear portion), and the other is solved explicitly.

Because of the complexities of implicit methods, very few attempts have been made in using this type of methods. Stalidis et al. [13] used an implicit finite difference method, but applied to the Monodomain model, and similarly in Chudin et al. [4]. Pormann [11] incorporates a similar approach, but using a matrix splitting procedure. Pennacchio and Simoncini [10] introduce the use of an algebraic semi-implicit form which is applied to a system with some degree of anisotropy. Lines [8] uses a similar operator splitting technique, also semi-implicit.

As mentioned above, the existing methods suffer from severe limitations because they use either explicit or semi-implicit approaches for numerically solving the problem at hand. In our case, we propose to use a fully implicit method which does not imply the cost and penalties of conventional fully-implicit methods; it is the Newton-Krylov-Schwarz method based on the Domain Decomposition technique.

\subsection{Discretization}

In order to numerically solve the system of time-dependent nonlinear partial differential equations which represent the Bidomain Model, we first need to discretise them. We choose to use a fully implicit finite difference scheme. We use a centered, finite difference 5-point stencil for the space variables, and backward Euler for the time variable. 


\subsection{Newton Method}

Once we have the discretized version, we need to put our system of nonlinear equations into the form $F(u)=0$ for each time step, where

$$
\left\{\begin{array}{c}
F=\left(f_{1}(v), f_{2}(v), \ldots, f_{M}(v)\right) \\
v=\left(v_{11}^{n}, v_{12}^{n}, \ldots, v_{m m}^{n}\right)
\end{array}\right.
$$

This allows us to use Newton's method to solve it [2]. $v$ here represents our state vector (in the case of the heart excitation process, we can assume $v$ represents the values of the potential at each of the points of our domain).

Some particular characteristics of the system of equations for the given problem need to be mentioned: first, the Jacobian matrix is very sparse; this makes the problem a lot more tractable, in terms of both storage and computing time. Second, the type of equations makes it relatively simple to compute the Jacobian elements directly from our given function values in $F(v)$.

At each Newton step, we need to solve the following Linear system of equations:

$$
J\left(v^{k}\right) \Delta v_{k}=-F\left(v^{k}\right)
$$

In our particular case, we choose to use the Newton-Krylov technique where each of the individual iterations is solved inexactly, but still having the overall process retain the convergence properties. The idea is to have fewer iterations at each step, specially at the beginning steps where we probably are still far from the solution. As the iterates get closer to the solution, then it might be more necessary, and more productive to have more iterations at each of these linear solver steps.

\subsection{Krylov Method}

In particular, we choose the Newton-Krylov method for solving (inexactly) the Linear system at each iteration step. When our Jacobian matrix $J$ is s.p.d. , we can compute the iterates by using the Conjugate Gradient method. In our particular research, we need to resort to the use of the GMRES technique in cases where our Jacobian matrix is no longer symmetric; for example, when the bidomain model is allowed to have some complexity (as some degree of anisotropy and inhomogeneity). We improve the efficiency and robustness by means of a Preconditioning technique; in particular, we use the Additive Schwarz method as preconditioner [7]. This preconditioning method is chosen due to its high convergence rate, and equally important, due to its extremely good parallel performance; based on the Domain Decomposition criteria, this method tries to fully utilize the parallel capabilities of a given system to its fullest potential. 


\subsection{Additive Schwarz Preconditioner}

The Schwarz preconditioning is a Domain Decomposition method [] which exhibits ideal features. First, the idea is to subdivide our domain into smaller subdomains, and to solve simpler and smaller problems in each of these subdomains, and then combine these solutions in order to obtain the solution to our more general and complicated problem. Second, parallelism plays a very important factor in these type of decomposition techniques since if the problem is properly subdivided we could fully utilize the parallel capabilities of our machines by working on all subdomains in parallel.

We then solve the problem at each subdomain, and only update the general solution once all the subdomains have been solved for. By using the Domain Decomposition method, we extract from our system of equations only the portion that corresponds to each subdomain (or processor), and compute in parallel

$$
M_{i}=L_{i} U_{i}
$$

in the sense of an incomplete factorization.

At the subdomain level, we resort to an inexact solver such as the ILU (Incomplete LU decomposition) [12] technique since it can reduce considerably the time and required storage of each iteration. Once we solve this subdomain problem using the ILU technique, the new iterate is found by combining the solutions at each of the subdomains.

\subsection{Algorithm}

In summary, we present here the basic steps for the algorithm we use in solving the given bidomain equations:

- Iterate through time:

1. Using Newton method to solve $F(v)=0$

2. At each Newton step:

- Solve the linear system : $J\left(v_{k}\right) \delta u=-F\left(v_{k}\right)$ with Additive Schwarz combined with Conjugate Gradient (or GMRES)

- Use ILU to solve for the subdomain problem in Schwarz preconditioning

- Update Jacobian and the Function

3. Go to next Newton step (or stop)

- Go to next time step (or stop) 


\section{Computational Results}

The experiments were carried out to implement and solve for the Bidomain equations, in the case that various degrees of anisotropy are included. We first define our two-dimensional domain as a square representing a thin slab of the heart tissue, with dimensions $1 \mathrm{~cm}$ by $1 \mathrm{~cm}$. The uniform grid is made up of 64 nodes on each direction, which gives a total of 8192 equations, since there are two degrees of freedom per node (one for the transmembrane potential, and one for the extracellular potential). We maintain this size to be comparable to the experiments performed in [10], and in [1]. The simulation was initiated by applying an initial current of $0.8 \mathrm{~A} / \mathrm{cm}^{3}$ for a duration of 0.5 msec. as used in [10], and only to a portion of our domain (a small semi-circle around the center of the left edge).

Other parameters were chosen from the literature [10], and are summarized below. Similarly to the author, we have restricted our experiments to one instant of time; therefore, we assume that the information from previous time steps is available.

Table 1. Parameters for Ionic current and others

\begin{tabular}{llr}
\hline$\chi=1000 \mathrm{~cm}^{-1}$ & $A=0.04 \mathrm{mS} / \mathrm{cm}^{2}$ & $c_{m}=0.8 \mu \mathrm{F} / \mathrm{cm}^{2}$ \\
$I_{a p p}=0.8 \mathrm{~A} / \mathrm{cm}^{3}$ & $V_{p}=100 \mathrm{mV}$ & $V_{t}=10 \mathrm{mV}$ \\
\hline
\end{tabular}

For the anisotropy, we assume conductivity coefficients in our matrix $D$ to be along and across the tissue fibers; we used three different set of values which indicate various degrees of anisotropy, and different ratios between the conductivity values in the two domains. We summarize here the values taken from [10], [8], and [1].

Table 2. Conductivity values and Anisotropy ratios

\begin{tabular}{llr}
\hline \hline & & \\
& & \\
& & \\
$\sigma_{l}^{i}=2.0 \mathrm{mS} / \mathrm{cm}$ & $\sigma_{l}^{e}=2.5 \mathrm{mS} / \mathrm{cm}$ & ratio $=0.8$ \\
$\sigma_{t}^{i}=0.416 \mathrm{mS} / \mathrm{cm}$ & $\sigma_{t}^{e}=1.25 \mathrm{mS} / \mathrm{cm}$ & ratio $=0.33$ \\
& 2. Lines $[8]$ & \\
& & \\
$\sigma_{l}^{i}=3.0 \mathrm{mS} / \mathrm{cm}$ & $\sigma_{l}^{e}=2.0 \mathrm{mS} / \mathrm{cm}$ & ratio $=1.5$ \\
$\sigma_{t}^{i}=0.31525 \mathrm{mS} / \mathrm{cm}$ & $\sigma_{t}^{e}=1.3514 \mathrm{mS} / \mathrm{cm}$ & ratio $=0.23$ \\
& 3. Bogar $[1]$ & \\
& & \\
$\sigma_{l}^{i}=4.0 \mathrm{mS} / \mathrm{cm}$ & $\sigma_{l}^{e}=4.0 \mathrm{mS} / \mathrm{cm}$ & ratio $=1.0$ \\
$\sigma_{t}^{i}=1.0 \mathrm{mS} / \mathrm{cm}$ & $\sigma_{t}^{e}=4.0 \mathrm{mS} / \mathrm{cm}$ & ratio $=0.25$ \\
& & \\
\hline \hline
\end{tabular}


We have previously presented results where we use the Newton-Krylov-Schwarz approach for the simplest cases, the Monodomain case in [3], and for the quasiIsotropic case in [9], where the ratios are equal, and therefore the equations simplify and become basically the Monodomain equation.

The results that we have obtained are summarized below, and they indicate that the various degrees of anisotropy can affect slightly the rate of convergence of the linear as well as the nonlinear equations we are solving at each Newton step. In general, we have not put major emphasis on getting the linear iterations to totally converge since in our Krylov method, we allow for some approximation. The key portion is to obtain convergence in the nonlinear portion of the method, and we see that we are obtaining such convergence without having to execute a large number of nonlinear iterations.

Table 3. Number of iterations (linear and nonlinear)

$\begin{array}{ccc}\text { Case } & \text { AverageLinearIterations } & \text { AverageNonlinearIterations } \\ 1 & 100 & 7 \\ 2 & 100 & 10 \\ 3 & 100 & 20\end{array}$

\section{Conclusion}

The preliminary results of applying the proposed Newton-Krylov-Schwarz algorithm to solve the more complex model of the electrical activity of the heart, indicate that this method seem capable of dealing with more complicated structure in our model, by incorporating anisotropy and applying the technique to the truly Bidomain model. These results extend from our previous work on the simpler models, and considering the parallel capabilities of this Domain Decomposition technique, major progress is expected when the technique is implemented and extended to larger problems.

\section{References}

[1] K. Bogar. A Semi-Implicit Time Scheme for the Study of Action Potential Propagation in the Bidomain Model. PhD thesis, University of Utah, 1999.

[2] R. Burden and J. Faires. Numerical Analysis. Prindle, Weber and Schmidt, 1985. 
[3] X. Cai and M. Murillo. Parallel performance of a newton-krylov-schwarz solver in a ed numerical simulation of the excitation process in the heart. Eight SIAM Conference on Parallel Processing for Scientific Computing, page 25, 1997.

[4] E. Chudin et al. Wave propagation in cardiac tissue and effects of intracellular calcium dynamics (computer simulation study). Progress in Biophysics and Molecular Biology, 69:225-236, 1998.

[5] D. DiFrancesco and D. Noble. A model of cardiac electrical activity incorporating ionic pumps and concentration changes. Philosophical Transactions of the Royal Society of London B, 307:353-398, 1985.

[6] P. Grottum, A. Tveito, and D. Zych. Heart - numerical simulation of the excitation process in the human heart, 1995. http://www.oslo.sintef.no/adv/33/3340/diffpack.

[7] N. Hooke, C. Henriquez, P. Lanzkron, and D. Rose. Linear algebraic transformations of the bidomain equations: Implications for numerical methods. Mathematical Biosciences, 120:127-145, 1994.

[8] D. Keyes, Y. Saad, and D. Truhlar. Domain-Based Parallelism and Problem Decomposition Methods in Computational Science and Engineering. SIAM, 1995.

[9] G. T. Lines. Simulating the Electrical Activity of the Heart. PhD thesis, University of Oslo, 1999.

[10] M. Murillo and X. Cai. Parallel algorithm and software for solving timedependent nonlinear bidomain equations. First SIAM Conference on Computational Science and Engineering, page 69, 2000.

[11] M. Pennacchio and V. Simoncini. Efficient algebraic solution of reactiondiffusion sysems for the cardiac excitation process. preprint, pages 1-19, 1999.

[12] J. Pormann. A Modular Simulation System for the Bidomain Equations. PhD thesis, Duke University, 1999.

[13] Y. Saad. Iterative Methods for Sparse Linear Systems. PWS Kent, 1995.

[14] G. Stalidis et al. Application of a 3-d ischemic heart model derived from mri data to the simulation of the electrical activity of the heart. Computers in Cardiology, pages 329-332, 1996.

[15] S. Veronese and H. Othmer. A computational study of wave propagation in a model for anisotropic cardiac ventricular tissue. Lecture Notes in Computer Science, 919:248-253, 1995. 